PSFC/JA-09-18

\title{
Full wave simulations of lower hybrid wave propagation in tokamaks
}

\author{
J. C. Wright*, P. T. Bonoli*, C. K. Phillips ${ }^{\dagger}$, \\ E. Valeo ${ }^{\dagger}$ and $R$. W. Harvey** \\ *MIT - Plasma Science and Fusion Center Cambridge, MA 02139 \\ ${ }^{\dagger}$ Princeton Plasma Physics Laboratory - Princeton, New Jersey, USA \\ **Comp-X - Del Mar, CA 92014
}

July 2009

\section{Plasma Science and Fusion Center Massachusetts Institute of Technology Cambridge MA 02139 USA}

This work was supported by the U.S. Department of Energy, Contract No. DE-FCO201ER54648. Reproduction, translation, publication, use and disposal, in whole or in part, by or for the United States government is permitted 


\title{
Full wave simulations of lower hybrid wave propagation in tokamaks
}

\author{
J. C. Wright*, P. T. Bonoli*, C. K. Phillips ${ }^{\dagger}$, E. Valeo ${ }^{\dagger}$ and R. W. Harvey** \\ * MIT - Plasma Science and Fusion Center Cambridge, MA 02139 \\ ${ }^{\dagger}$ Princeton Plasma Physics Laboratory - Princeton, New Jersey, USA \\ ${ }^{* *}$ Comp-X - Del Mar, CA 92014
}

\begin{abstract}
Lower hybrid ( $\mathrm{LH})$ waves have the attractive property of damping strongly via electron Landau resonance on relatively fast tail electrons at $(2.5--3) \times v_{\mathrm{te}}$, where $v_{\mathrm{te}} \equiv\left(2 T_{e} / m_{e}\right)^{1 / 2}$ is the electron thermal speed. Consequently these waves are well-suited to driving current in the plasma periphery where the electron temperature is lower, making LH current drive (LHCD) a promising technique for off-axis $(r / a \geq 0.60)$ current profile control in reactor grade plasmas. Established techniques for computing wave propagation and absorption use WKB expansions with non-Maxwellian self-consistent distributions. Higher order WKB expansions have shown some effects from diffraction on the parallel wavenumber evolution and consequently on the damping location.

In typical plasma conditions with electron densities of several $10^{19} \mathrm{~m}^{-3}$ and toroidal magnetic fields strengths of 4 Telsa, the perpendicular wavelength is of the order of $1 \mathrm{~mm}$ and the parallel wavelength is of the order of $1 \mathrm{~cm}$. Even in a relatively small device such as Alcator C-Mod with a minor radius of $22 \mathrm{~cm}$, the number of wavelengths that must be resolved requires large amounts of computational resources for the full wave treatment. These requirements are met with a massively parallel version of the TORIC full wave code that has been adapted specifically for the simulation of LH waves [J. C. Wright, et al., Commun. Comput. Phys., 4, 545 (2008), J. C. Wright, et al., Phys. Plasmas 16 July (2009)]. This model accurately represents the effects of focusing and diffraction that occur in LH propagation. It is also coupled with a Fokker-Planck solver, CQL3D, to provide self-consistent distribution functions for the plasma dielectric as well as a synthetic HXR diagnostic for direct comparisons with experimental measurements of LH waves.

The wave solutions from the TORIC zero FLR model will be compared to the results from ray tracing from the GENRAY/CQL3D code via the synthetic HXR diagnostic and power deposition.
\end{abstract}

\section{INTRODUCTION}

Electromagnetic waves in the lower hybrid range of frequencies (LHRF), are characterized by a frequency approximately at the geometric mean of the ion and electron fundamental cyclotron frequencies, $\omega_{\mathrm{LH}} \approx \sqrt{\Omega_{\mathrm{ci}} \Omega_{\mathrm{ce}}}$. They have the attractive property of damping strongly via electron Landau resonance on relatively fast tail electrons at 2.5 $v_{\mathrm{te}}$, where $v_{\mathrm{te}}=\left(2 T_{e} / m_{e}\right)^{1 / 2}$ is the electron thermal speed. Consequently these waves are well-suited to off-axis $(r / a>0.60)$ current profile control in reactor grade plasmas with high electron temperature.

At these frequencies, the wavelengths are on the order of a few millimeters at values of the dielectric constant, $\left(\omega_{\mathrm{pe}} / \Omega_{\mathrm{ce}}\right)^{2}$, for typical parameters of fusion research devices such as Alcator C-Mod and the proposed International Tokamak Experimental Reactor (ITER), where they have been proposed as a method of edge current profile control [1]. 
Three waves are supported by the plasma at this frequency range: the slow lower hybrid wave (usually the one referred to as the lower hybrid wave, the fast lower hybrid wave (or Whistler wave), and the ion plasma wave which is supported by finite ion Larmor radius effects. As explained in Section 2. of [2], at plasma parameters of experimental interest that are used in the simulations presented in this paper, the ion plasma wave is strongly evanescent, and so only two waves are supported. The TORIC-LH algorithm only supports the fast and slow propagating modes [3].

Ray tracing has been the preferred method for lower hybrid models both because resolution requirements for full wave calculations have been prohibitive and because the small ratio of wavelength to system size has well suited the eikonal approximation. However, the geometric optics WKB approximation does have trouble capturing some effects. It cannot treat mode conversion physics, but see [Richardson, this proceedings] for attempts to deal with this within an extended WKB framework. Caustics also cause some difficulty for ray tracing, but Maslov techniques[4] provide a way of avoiding the vanishing wavenumbers at caustic surfaces which would violate WKB. Finally, diffraction of the rays - a possible mechanism of spectral broadening - cannot be treated by ray tracing, though hybrid full wave-WKB techniques have been tried[5] and found enhanced spectral broadening due to diffraction especially at caustic surfaces.

In this paper, we apply the previously developed full wave calculations of lower hybrid waves in toroidal geometry to an Alcator C-Mod lower hybrid experiment and also compare results with ray tracing calculations. In this experiment, ray tracing predicts significantly narrower current deposition than is measured when the $n_{\|}$is low and the absorption is weak [6], and so we are motivated to explore full wave effects.

\section{THE WAVE EQUATION AND PLASMA MODEL}

The wave equation that results from the linearized Maxwell-Boltzmann system [7, 8] is given in Eq. (1). The plasma response embodied in the term $\mathbf{J}^{P}$ in Eq. (2). We have formulated the plasma conductivity, $\sigma$, for the LHRF in which the lower hybrid

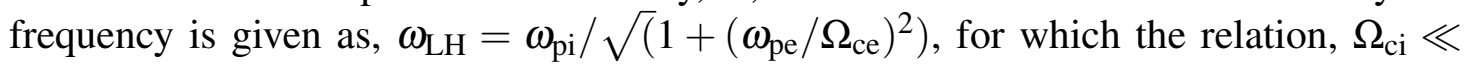
$\omega \sim \omega_{\mathrm{LH}} \ll \Omega_{\mathrm{ce}}$ holds.

$$
\begin{gathered}
\nabla \times \nabla \times \mathbf{E}=\frac{\omega^{2}}{c^{2}}\left\{\mathbf{E}+\frac{4 \pi i}{\omega}\left(\mathbf{J}^{P}+\mathbf{J}^{A}\right)\right\} \\
\mathbf{J}^{P}=\stackrel{\leftrightarrow}{\sigma}\left[f_{0}\left(\mathbf{x}, \mathbf{v}_{\perp}, v_{\|}\right)\right] \cdot \mathbf{E}
\end{gathered}
$$

In this range of frequencies, the ions are unmagnetized and the electrons are strongly magnetized $\left[\left(k_{\perp} \rho_{\mathrm{e}}\right)^{2} \ll 1\right]$. Thus, we no longer need the ion finite Larmor radius (FLR)

effects and the LH plasma dielectric, $\overleftrightarrow{\varepsilon} \equiv \overleftrightarrow{1}+\frac{4 \pi \mathrm{i}}{\omega} \overleftrightarrow{\sigma}$, simplifies to the zero FLR contributions: 


$$
\begin{aligned}
\overleftrightarrow{\sigma} \cdot \mathbf{E} & =S \mathbf{E}_{\perp}+\mathrm{i} D\left(\mathbf{b} \times \mathbf{E}_{\perp}\right)+P E_{\zeta} \mathbf{b} \\
S & \approx 1+\frac{\omega_{\mathrm{pe}}^{2}}{\Omega_{\mathrm{ce}}^{2}}-\frac{\omega_{\mathrm{pi}}^{2}}{\omega^{2}} \approx 1 \\
D & \approx-\frac{\omega_{\mathrm{pe}}^{2}}{\omega^{2}} \frac{\omega}{\Omega_{\mathrm{ce}}}+\frac{\omega_{\mathrm{pi}}^{2}}{\omega^{2}} \frac{\Omega_{\mathrm{ci}}}{\omega} \approx-\frac{\omega_{\mathrm{pe}}^{2}}{\omega \Omega_{\mathrm{ce}}} \\
P & =1-\frac{\omega_{\mathrm{pe}}^{2}}{\omega^{2}}-\frac{\omega_{\mathrm{pi}}^{2}}{\omega^{2}} \approx \frac{\omega_{\mathrm{pe}}^{2}}{\omega^{2}}
\end{aligned}
$$

where $S, D$, and $P$, are the Stix cold plasma dielectric elements in the LHRF for the normal, co-normal, and parallel directions [9]. The neglected FLR corrections to Eq. (3) support the mode converted ion plasma wave. Since this wave does not propagate for plasmas of experimental interest in which $\omega / \omega_{\mathrm{LH}}>2$ [10], we may neglect these corrections in the following analysis, and thus there are only two propagating modes, the fast electromagnetic LH branch that damps via electron Landau damping (ELD) and transit time magnetic pumping (TTMP) and the slow electrostatic LH branch that damps via ELD. Note, that although the FLR terms play no role, thermal effects are retained through the plasma dispersion function contributions to the ELD and TTMP damping in the plasma model. If the FLR electron corrections to $S$ and $D$ that represent transit time magnetic pumping are retained, a numerical mode is created [3] that corrupts the solution. The magnitude of these terms in the LHRF makes them entirely negligible and so they are dropped.

\section{SELF-CONSISTENT COUPLING}

Lower hybrid waves operate at high phase velocity for the most efficient current drive but must avoid the accessibility limit [10] that occurs at very high phase velocities $\left(v_{\phi} \equiv c / n_{\|}\right)$with a parallel index, $n_{\|}$, less than $n_{a} \approx \frac{\omega_{\mathrm{pe}}}{\Omega_{\mathrm{ce}}}+1 \approx 1.4$ for Alcator C-Mod parameters $\left(B_{0} 4 T, n_{e 0} 4 \times 10^{19} / \mathrm{m}^{3}\right)$. The interaction of the waves far out on the tail of the distribution, $f_{e 0}(v)$, creates a non-Maxwellian plateau that must be modeled selfconsistently. The evolution of $f_{e 0}(v)$ is described by the bounce averaged Fokker-Planck equation,

$$
\frac{\partial}{\partial t}\left(\lambda f_{e 0}\right)=\nabla_{u_{0}} \cdot \Gamma_{u_{0}}+\langle\langle R\rangle\rangle+\langle\langle S\rangle\rangle
$$

where $\langle\langle R\rangle\rangle$ is the bounce and gyro averaged collision operator and $\langle\langle S\rangle\rangle$ is the averaged wave induced velocity space diffusion and both are linear operators on $f_{e 0}(v)$, and $\lambda$ is the product of the midplane parallel velocity, $v_{\| 0}$, and the orbit period or bounce time.

To self-consistently determine the electron distribution and the damping, we iterate between the full wave TORIC code and the Fokker-Planck CQL3D code [11]. The coupling between the two codes takes place through the RF induced quasilinear diffusion, $\langle\langle S\rangle\rangle$, in velocity space in CQL3D and the use of a non-Maxwellian parallel dielectric 
in TORIC. In LHRF, the formulation of the wave induced diffusion tensor only has contributions from the parallel-parallel term, expressed as $\delta B$ in work by Harvey and McCoy [11] and which is a relativistic generalization of the Kennel and Engelmann [12] operator. To account for interference effects between the wave fields we adapt the expressions from Wright [13] for the quasilinear diffusion of electron in the ICRF to the LHRF resulting in:

$$
\lambda\langle\delta B\rangle=v_{\| 0} \oint \frac{d \ell}{\left|v_{\|}\right|} \frac{u_{\|}^{4} E_{\|}^{\left(m_{1}\right)} E_{\|}^{*\left(m_{2}\right)}}{\gamma^{2}\left(\omega / k_{\|_{1}}\right)\left(\omega / k_{\|_{2}}\right)} e^{i \theta\left(m_{1}-m_{2}\right)} \delta\left(\omega-v_{\|} k_{\|}\right) J_{0}\left(k_{\perp} u_{\perp} / \Omega_{\mathrm{ce}}\right),
$$

in which we have kept only the zeroth order electron FLR terms, $\gamma$ is the relativistic Lorentz factor, $\delta$ is the usual delta function resonance term, $J_{0}$ is the Bessel function of order zero, and $u \equiv p / m=\gamma v$ is the relativistic particle momentum per rest mass. The Bessel term accounts for the reduction of diffusion at high perpendicular energies and is evaluated using the LH slow wave root. This diffusion expression is evaluated and bounce averaged assuming zero banana width electrons in a post-processing mode of TORIC. It is then mapped onto the polar velocity space mesh and stored in a format used by CQL 3D. CQL 3D uses this diffusion coefficient scaled to couple the desired amount of power (since the wave solution is linear and is done for a unit applied parallel electric field of $1 \mathrm{~V} / \mathrm{m}$ ). This is necessary because the full wave code is linear and the amount of power coupled by the waveguide depends on the plasma loading as well as the electric field specified at the guide. The scale factor needs only to be adjusted at the beginning of the iteration process. The loop is closed by the use of the CQL3D generated electron distribution function, $f_{e 0}(\mathbf{v})$, in the next TORIC run. Because LH interacts with electrons at parallel speeds greater the 2.5 times the thermal velocity, only the anti-Hermitian part of the parallel dielectric is affected significantly. Therefore, in Eq. (3) only damping in $P$ is modified and it is proportional to the parallel velocity space derivative of $f_{e 0}(\mathbf{v})$ [9]. We use the technique of Valeo [14] with which we also verified by direct evaluation of the principal value that the real dielectric was not modified by non-Maxwellian features, only the damping. The two codes are iterated in this coupled fashion until the power deposition no longer changes and agrees between the two codes.

For simplicity we take the Lorentz factor $\gamma=1$ and so $u_{\|}=v_{\|}$. The bounce average introduces structure in velocity space through the mirror effect which couples the parallel motion to the perpendicular through conservation of the magnetic moment, $\mu=v_{\perp}^{2} / B$ and energy: $u_{\|}(\ell)=u_{\|}(0) \sqrt{B(\ell) / B(0)}$. This effect is apparent in Figure 1 .

\section{APPLICATION TO ALCATOR C-MOD}

We now apply this new algorithm to recent lower hybrid experiments on Alcator C-Mod experiment [A. Schmidt, this proceedings]. In discharge 1060728011 at $1 \mathrm{~s}$, the loop voltage was maintained at $-0.2 \mathrm{~V}$ out of $1.0 \mathrm{~V}$ by lower hybrid current drive. The parameters of the simulation are given in Figure 2 and correspond to a scenario in which the lower hybrid waves are accessible to the center of the device. 

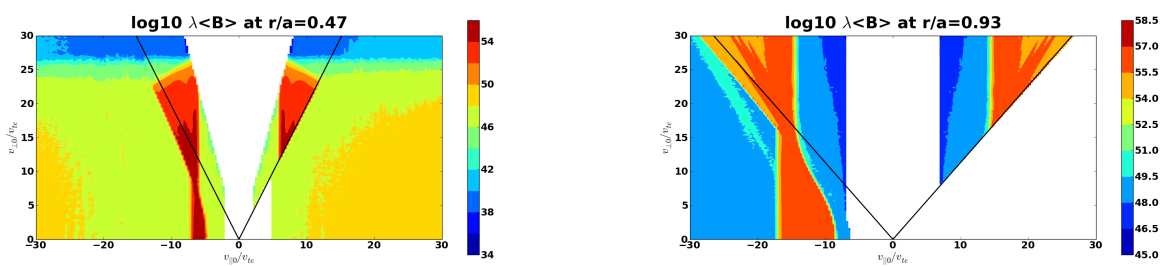

FIGURE 1. Bounce averaged quasilinear diffusion at two flux surface. Nearly trapped electrons spend more time in resonance at their turning points causing the contours to bend towards the passing trapped boundary marked in solid black lines.
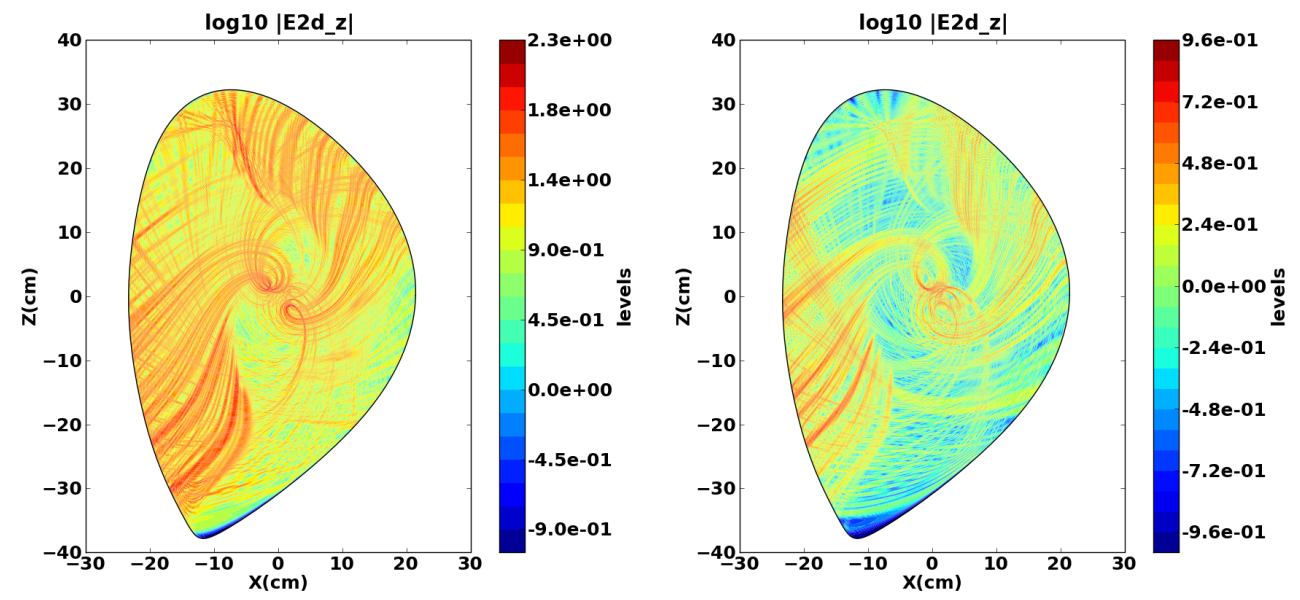

FIGURE 2. Full wave simulation of lower hybrid waves in Alcator C-Mod experiment. Left figure is the first run and the right figure is after three iterations with the Fokker-Planck code. There are four $6 \mathrm{~cm}$ high waveguides on the low field (right) side. Parameters of runs: $\left(980 N_{\psi} x 2048 N_{\theta}\right)$ D plasma, $n_{\|}=-1.55$, $\mathrm{f}=4.6 \mathrm{GHz} \mathrm{B} \mathrm{B}_{0}=5.36 \mathrm{~T}, \mathrm{~T}_{e}=2.33 \mathrm{keV}, \mathrm{T}_{i}=1.0 \mathrm{keV} n_{e}(0)=0.7 \times 10^{20} m^{-3}, \mathrm{I}=545 \mathrm{kA}$

Full wave simulation of this shot shows that for damping on a Maxwellian distribution, the fields are space filling and are amplified by a factor of $\approx 200$ from the applied field $(1 \mathrm{~V} / \mathrm{m})$ because of the cavity effect in weak damping. The right plot in Figure 2 is of the contours of the parallel electric field after iterating to self-consistency with the Fokker-Planck code.

We expect some enhanced spectral broadening and acceleration of the filling of the spectral gap in the presence of diffraction in the full wave solver. The primary sources of this diffraction are caustic surfaces and scattering during reflections from the plasma cutoff. However, in the case of damping on a Maxwellian, we see no indication of this in the power deposition. That is, the damping profiles from the full wave do not show a significant difference from those of ray tracing which lacks these effects.

By observing the evolution of non-Maxwellian features in the electron distribution we can see direct evidence of the spectral broadening. We have used the electric fields from the full wave solution shown in Figure 2(left panel) to formulate the RF quasilinear diffusion coefficient. This was then employed in the Fokker-Planck code CQL $3 D$ 

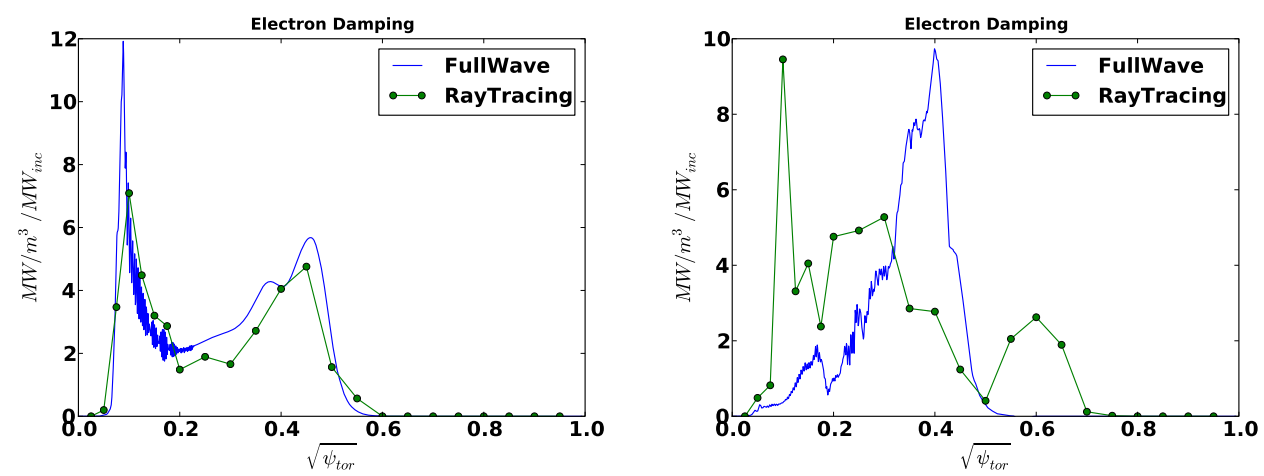

FIGURE 3. Power deposition from the full wave code compared with ray tracing with linear (Maxwellian) damping (left plot) and non-linear damping (right plot). Plots are from the same cases as in Figure 2.

to evolve the electron distribution function. In the right panel of Figure 2, the electric fields are less intense due to stronger absorption. In the left panel of Figure 4, we see the formation of a quasilinear plateau clearly at a pitch angle of $\theta=0$ at a flux surface of $r / a \sim 0.5$ located in the region of power absorption. The plateau extends in a range of parallel velocities corresponding to $n_{\|}=[1.4,5]([0.7,0.3]$ on the relativistic velocity scale in the plot) demonstrating clear evidence of downshift of the phase velocity from the launched $n_{\|}$of -1.55 to fill the spectral gap. The right panel of Figure 4 shows a comparison of the hard X-ray (HXR) signal generated by this distribution function using a synthetic HXR diagnostic that mimics the experimental measurement. The direct comparison with experimental measurements avoids any necessity of inverting experimental data and introducing additional uncertainties. The simulated measurement shows that the full wave approach predicts a narrower depostion profile that is experimentally detected. In addition, the computed signal is too weak by about a factor of 4 . We speculate that full relativistic treatment of the dielectric in the full wave code and the inclusion of a finite toroidal antenna spectrum with the concomitant non-linear coupling of toroidal modes may account for these discrepancies.

The damping from the quasilinear modifications of $f_{e}(\mathbf{v})$ have a strong effect on the convergence of the spectral expansion. In the left panel of Figure 5, the power spectrum of the solution from Figure 2 demonstrates strong convergence even at outer flux surfaces. With linear damping, $4096 \theta$ mesh points are needed for a converged solution. The meaning of such convergence is questionable as well since the distribution function does not remain Maxwellian and results in more growth of the fields in the plasma due to the previously discussed cavity effect. At $2048 \theta$ mesh points the solution is only converged to about a radius of $r / a \approx 0.6$ as the damping reduces with temperature at outer flux surfaces and the contribution of the poloidal mode number to $n_{\|}$is reduced by the safety factor at larger radii limiting the amount of downshift in the phase velocity possible. The establishment of a quasilinear plateau in the self-consistent simulations converges the wave solution at 2048 theta mesh points (1023 poloidal modes.) The energy in the largest magnitude poloidal modes is orders of magnitude lower than the peaks in the spectrum at each flux surface - this is a good measure of convergence when 

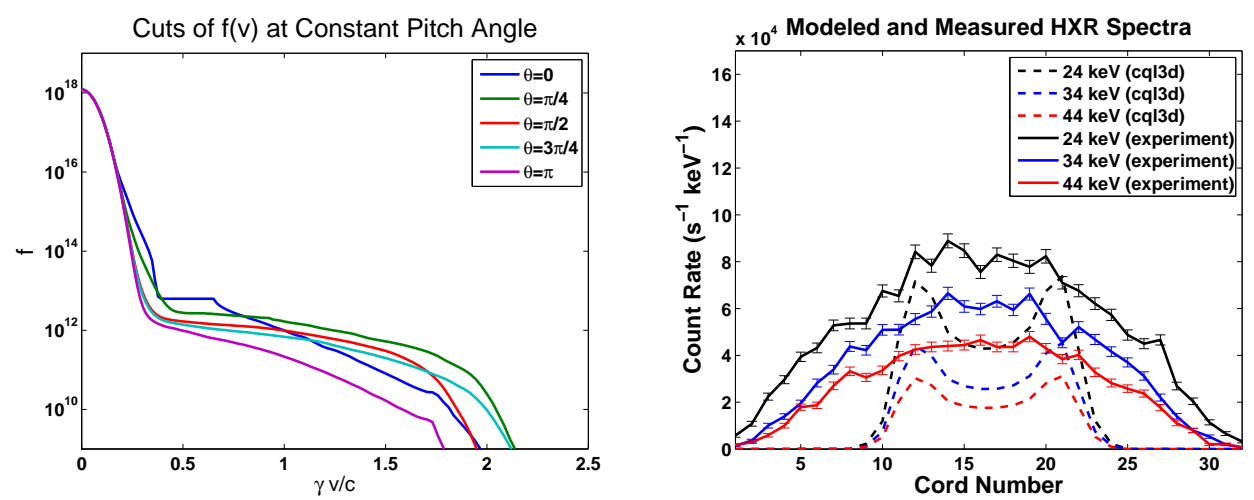

FIGURE 4. (Left plot) Electron distribution response to the LH waves' quasilinear flux generated from the fields in Figure 2. Plots of the distribution function versus relativistic velocity measure for several different pitch angles, $\theta$, at a flux surface of $r / a \sim 0.50$ in the multi-pass case. Parameters are the same as listed in Figure 2. $650 \mathrm{~kW}$ of power were coupled in this case. The right plot compares the synthetic HXR signal with the experimental one. Cord numbers correspond to different lines of site in the diagnostic with cord 16 being a midplane line of sight.
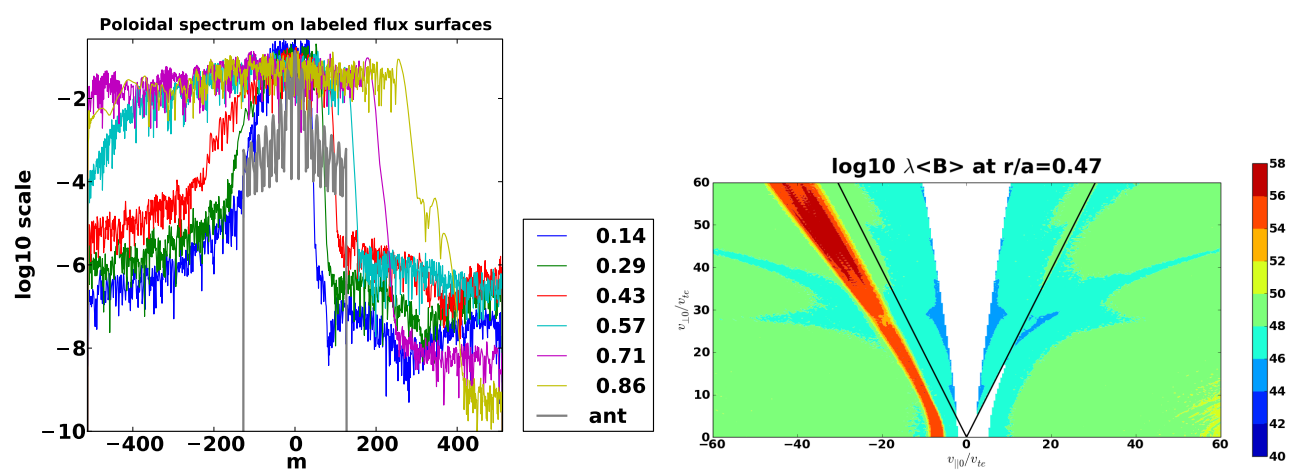

FIGURE 5. (Left panel) The poloidal power spectrum plotted for several flux surfaces and the waveguide surface for the parameters in Figure 2. (Right panel) The quasilinear diffusion coefficient including relativistic effects.

using a complete orthogonal basis set. We also observe the asymmetric nature of the spectrum. Noting that the poloidal width of the wave spectrum is related to the width of the wavenumber spectrum by $\delta n_{\|} / n_{\| 0} \sim \delta m / q n_{\phi}$ we observe that for the value of $n_{\| 0}=-1.55$, positive poloidal mode numbers decrease the magnitude of the local value of $n_{\|}$bringing the value closer to the accessibility limit. Once reached, we see the sharp and continuous exponential drop in amplitude. For negative poloidal modes, the phase velocity is shifted towards the bulk electrons where damping is strong and we see the more structured decrease in the spectrum. 


\section{CONCLUSIONS}

The necessary algorithms for solving full wave dispersion in realistic toroidal geometries have been developed by the RF community over the past few decades. We have applied these to the LHRF for a discharge in Alcator C-Mod. Full wave calculations have similar features to ray tracing with both showing the formation of caustics around the axis and similar power deposition in linear damping. Self-consistent non-linear simulations show differences between full wave and ray tracing and also with experiment. Non-linear coupling of toroidal modes and a full relativistic treatment in the full wave code of the dielectric are effects that have yet to be tested that may explain the discrepancies with experiment.

\section{ACKNOWLEDGMENTS}

All simulation presented in this paper were performed on the MIT-PSFC Opteron/Infiniband cluster, Loki. Research sponsored by the U.S. Department of Energy, under Contract No. DE-AC05-00OR22725.

\section{REFERENCES}

1. ITER Physics Expert Group on Energetic Particles, Heating and Current Drive and ITER Physics Basis Editors, Nucl. Fusion, 39, 2495-2540 (1999).

2. Wright, J. C., Bonoli, P. T., Brambilla, M., Azevedo, E. D., Berry, L. A., Batchelor, D. B., Jaeger, E. F., Carter, M. D., Phillips, C. K., Okuda, H., Harvey, R. W., Myra, J. R., D’Ippolito, D. A., and Smithe, D. N., "Full-wave Electromagnetic Field Simulations of Lower Hybrid Waves in Tokamaks," in 16th Topical Conference on Radio Frequency Power in Plasmas, edited by P. Bonoli and S. Wukitch, 787, American Institute of Physics, New York, 2005, p. 287.

3. Wright, J. C., Bonoli, P. T., Schmidt, A. E., Phillips, C. K., Valeo, E. J., Harvey, R. W., and Brambilla, M. A., Phys. Plasmas, 16, 0645xx (2009).

4. Chapman, C. H., and Keer, H., Stud. Geophys. Geo., 46, 615-649 (2004).

5. Pereverzev, G. V., Nucl. Fusion, 32, 1091-1107 (1992).

6. Bonoli, P. T., Hubbard, A. E., Ko, J., Parker, R., Schmidt, A. E., Wallace, G., Wright, J. C., the Alcator C-Mod Team, Wilson, J. R., Phillips, C. K., Scott, S., Valeo, E., and Harvey, R. W., Phys. Plasmas, 15, 056117 (2008).

7. Stix, T. H., Waves in Plasmas, chap. 10, p. 250, in [9] (1992).

8. Brambilla, M., Plasma Phys. Controlled Fusion, 31, 723-757 (1989).

9. Stix, T. H., Waves in Plasmas, American Institute of Physics, New York, 1992.

10. Bonoli, P., IEEE Trans. Plasma Sci., PS-12, 95-107 (1984).

11. Harvey, R. W., and McCoy, M. G., "The CQL3D Fokker-Planck Code," in Proc. of the IAEA Tech. Committee Meeting (Montreal,1992), IAEA, Institute of Physics Publishing; USDOC/NTIS Doc. DE93002962, Vienna, 1993, pp. 489-526.

12. Kennel, C. F., and Engelmann, F., Phys. Fluids, 9, 2377 (1966).

13. Wright, J. C., Phillips, C. K., and Bonoli, P. T., Nucl. Fusion, 37, 123-125 (1997).

14. Valeo, E. J., Phillips, C. K., Bonoli, P. T., and Wright, J. C., "Full-wave Simulations of LH wave propagation in toroidal plasma with non-Maxwellian electron distributions," in 17th Topical Conference on Radio Frequency Power in Plasmas, edited by P. Ryan and D. Rasmussen, 933, American Institute of Physics, New York, 2007, p. 297. 